\title{
Determination of erythrocyte sodium sensitivity in man
}

\author{
Hans Oberleithner • Marianne Wilhelmi
}

Received: 26 March 2013 /Revised: 27 April 2013 / Accepted: 28 April 2013 /Published online: 19 May 2013

(C) The Author(s) 2013. This article is published with open access at Springerlink.com

\begin{abstract}
Sodium buffer capacity of vascular endothelium depends on an endothelial glycocalyx rich in negatively charged heparan sulfate. It has been shown recently that after the mechanical interaction of blood with heparan sulfate-depleted endothelium, erythrocytes also lose this glycocalyx constituent. This observation led to the conclusion that the vascular sodium buffer capacity of an individual could be derived from a blood sample. A test system (salt blood test (SBT)) was developed based upon the sodium-dependent erythrocyte zeta potential. Erythrocyte sedimentation velocity was measured in isosmotic, biopolymer-supplemented electrolyte solutions of different sodium concentrations. Erythrocyte sodium sensitivity (ESS), inversely related to erythrocyte sodium buffer capacity, was expressed as the ratio of the erythrocyte sedimentation velocities of $150 \mathrm{mM}$ over $125 \mathrm{mM} \mathrm{Na}^{+}$ solutions ( $\mathrm{ESS}=\mathrm{Na}^{+}{ }_{150} / \mathrm{Na}^{+}{ }_{125}$ ). In 61 healthy individuals (mean age, $23 \pm 0.5$ years), ESS ranged between 2 and 8 . The mean value was $4.3 \pm 0.19$. The frequency distribution shows two peaks, one at about 3 and another one at about 5. To test whether ESS reflects changes of the endothelial glycocalyx, a cultured endothelial monolayer was exposed for 3 hours to a rhythmically moving blood layer (drag force experiment). When applying this procedure, we found that ESS was reduced by about $21 \%$ when the endothelium was pretreated for 4 days with the glycocalyx protective agent WS 1442. In conclusion, the SBT could possibly serve as an in vitro test system for the evaluation of erythrocyte/vascular salt sensitivity allowing follow-up measurements in the prevention and treatment of vascular dysfunctions.
\end{abstract}

H. Oberleithner $(\bowtie) \cdot$ M. Wilhelmi

Institute of Physiology II, University of Münster,

Robert-Koch-Str. 27b,

48149 Münster, Germany

e-mail: oberlei@uni-muenster.de
Keywords Erythrocyte glycocalyx $\cdot$ Heparan sulfate Endothelial glycocalyx $\cdot$ Red blood cell sedimentation velocity $\cdot$ Vascular sodium sensitivity

\section{Introduction}

At least one third of the world's population is supposed to be sensitive to salt [32], i.e., arterial hypertension occurs in response to the daily ingestion of large amounts of sodium, a habit widely spread in our societies [19]. Sodium is bound, osmotically inactive, to the negatively charged proteoglycans in the dermis [30] and endothelial glycocalyx (eGC) [1]. Sodium overload damages the eGC [24], thus diminishing endothelial sodium buffer capacity. Deterioration of the eGC allows sodium exiting the blood vessel lumen more readily via endothelial sodium channels [17, 26]. As a consequence, sodium spends more time in the extracellular space before being finally cleared by the kidneys $[16,22]$. This delayed sodium excretion leads in the long term to sodium deposition in the body, thereby damaging various tissues $[12,14]$. Based on this concept, the salt provocation test was developed which quantifies vascular sodium sensitivity in vivo [21]. Vascular sodium sensitivity, inversely related to endothelial sodium buffer capacity, characterizes the quality of the endothelial glycocalyx. Recently, it has been realized that the glycocalyx of erythrocytes (red blood cells (RBC)) reflects some specific surface properties of endothelial cells [20]. In more details, RBC allowing to physically interacting ("drag force treatment") with the endothelial surface in vitro acquire characteristics of the endothelial surface layer. For example, when the endothelial surface layer is experimentally depleted of negative charges - heparan sulfate shedding by the application of the enzyme heparinase - and then RBC allowed to interact for some hours, the RBC glycocalyx is found shed after such a drag force experiment [20]. This observation led to the conclusion that the vascular 
salt sensitivity could be indirectly measured by evaluating the erythrocyte salt sensitivity (ESS). A method (salt blood test (SBT)) was developed based on the sodium sensitivity of the RBC zeta potential $[9,10]$. As a proof of principle, the hawthorn-derived compound WS 1442 known to improve endothelial function, in particular the endothelial glycocalyx $[2,26]$, was applied in vitro to test whether the SBT "reports" such changes.

\section{Methods}

Principle and procedure of the SBT

Experimental steps 1-3 are documented in Fig. 1a-c. In detail, $2 \mathrm{ml}$ of blood is drawn from volunteers by venous puncture using heparinized monovettes (Sarstedt Company, Sarstedt, Germany). Blood is transferred in 2-ml plastic vials (Eppendorf AG, Hamburg, Germany) and centrifuged at $1,800 \times \mathrm{g}$ for $5 \mathrm{~min}$. Plasma and buffy coat are removed, and erythrocytes were washed two times in $30 \mathrm{ml}$ of HEPES-buffered (10 mM HEPES=2-(4-(2-hydroxyethyl)1-piperazinyl)-ethane sulfonic acid) sodium chloride (150 mM NaCl) including $1 \%$ bovine serum albumin (PAA Clone, Coelbe, Germany). This amount of serum is necessary for proper shielding of the glycocalyx. For a single measurement, $80 \mu \mathrm{l}$ of washed RBC is suspended in $120 \mu \mathrm{l} \mathrm{NaCl}$ solution (fixed hematocrit, 0.4 ) containing $3 \%$ dextran (Sigma 4486, MW 70,000 D). Dextran is adsorbed to the RBC surface triggering RBC aggregation (Fig. 1d, e). This phenomenon is called "macromolecular bridging," indicating that the adhesion forces between $\mathrm{RBC}$ overcome the electrostatic repulsive forces $[9,10]$. $\mathrm{NaCl}$ concentrations were varied between 50 and $150 \mathrm{mM}$ as appropriate. Sucrose at various amounts was added for maintaining constant osmolality. The test solutions containing varying $\mathrm{NaCl}$ concentrations, varying sucrose concentrations, and a fixed concentration of dextran (3\%) are called " $\mathrm{Na}^{+}$cocktails" throughout the paper. Hematocrit capillary tubes (Safecap P75-2000; length $75 \mathrm{~mm}$; Scholz Company, Neubiberg, Germany) were filled by capillary forces with the respective RBC suspensions as appropriate. Hematocrit capillary tubes, closed at the lower end, were put on stands in an upright position (Fig. 2). RBC sedimentation rates were measured at appropriate time intervals. The dimensionless ESS values of the individual blood samples were calculated as the ratio of the respective RBC sedimentation velocities in 150 and $125 \mathrm{mM} \mathrm{Na}^{+}$cocktail (ESS = $\mathrm{L}_{150} / \mathrm{L}_{125}$ ). It should be mentioned that a single ESS measurement needs only about $200 \mu \mathrm{l}$ blood which can be obtained from a puncture of the fingertip. The SBT was performed at ambient temperature. $\mathrm{pH}$ values of the $\mathrm{Na}^{+}$ cocktails were around 7.4 (due to the endogenous buffering power of hemoglobin at the fixed hematocrit of 0.4).

Drag force treatment

Endothelial monolayers (human Eahy629 cell line) were cultured for 4 days on the bottom of $75-\mathrm{cm}^{2}$ culture flasks $[4,15,18]$ in the absence (control) and presence of the vasoprotective Crataegus extract WS $1442(10 \mu \mathrm{g} / \mathrm{ml})$ [2]. WS 1442 is known to improve glycocalyx function in vitro [26]. After this period, the culture medium was replaced by whole blood ( $2.5 \mathrm{ml}$ per flask) taken from volunteers (heparinized monovettes; Sarstedt Company, Sarstedt, Germany). The flasks, mounted on a shaker $\left(4\right.$ shifts per min, $7^{\circ}$ angle), were placed in a cell culture incubator $\left(37^{\circ} \mathrm{C}, 5 \%\right.$ $\mathrm{CO}_{2}$ ) and rocked for $3 \mathrm{~h}$ so that the $\mathrm{RBC}$ could mechanically interact with the surface of the endothelial cell layer. This procedure named drag force treatment was described in detail recently [20].

\section{Results}

In contrast to the standard erythrocyte sedimentation rate (ESR) which is performed in whole blood and strongly dependent on any inflammation-relevant proteins in the plasma phase [27], the sedimentation rate used for ESS measurements is independent of any proteins, but strongly dependent on the $\mathrm{NaCl}$ concentration of the electrolyte solution in which the erythrocytes have been suspended. In particular, sedimentation rate depends on the RBC zeta potential [10] and, thus, on the RBC glycocalyx. Since $\mathrm{Na}^{+}$neutralizes the negative charges of the RBC surface, sedimentation rate reflects the $\mathrm{Na}^{+}$buffer capacity of the $\mathrm{RBC}$ glycocalyx. As mentioned above, dextran is needed in all solutions; otherwise, aggregation does not occur. In pilot studies, we found out that the most suitable molecular mass of the dextran is $70,000 \mathrm{D}$, and the most adequate concentration is $3 \%$. This is in good agreement with a previous study [10]. Figure 2 shows the time dependence of the RBC sedimentation rate. Initial sedimentation rates are quite linear, but they saturate later on. This is due to the fact that a hematocrit of 0.40 was used so that after long time periods (hours), virtually all RBC, not only the large RBC aggregates, concentrate in the lower part of the tube. Therefore, the 60 -min values were chosen for analysis. Figure 3 shows the dependence of the erythrocyte sedimentation on $\mathrm{NaCl}$ concentration. Ion strength, and in particular the cation $\mathrm{Na}^{+}$, determines sedimentation velocity. The reason why the 125 and $150 \mathrm{mM} \mathrm{Na}^{+}$concentrations have been chosen for the SBT were as follows: The RBC glycocalyx is treated as a $\mathrm{Na}^{+}$buffer. At too low $\mathrm{Na}^{+}$concentrations (below $100 \mathrm{mM}$ ), the negative charges of the RBC glycocalyx are heavily 

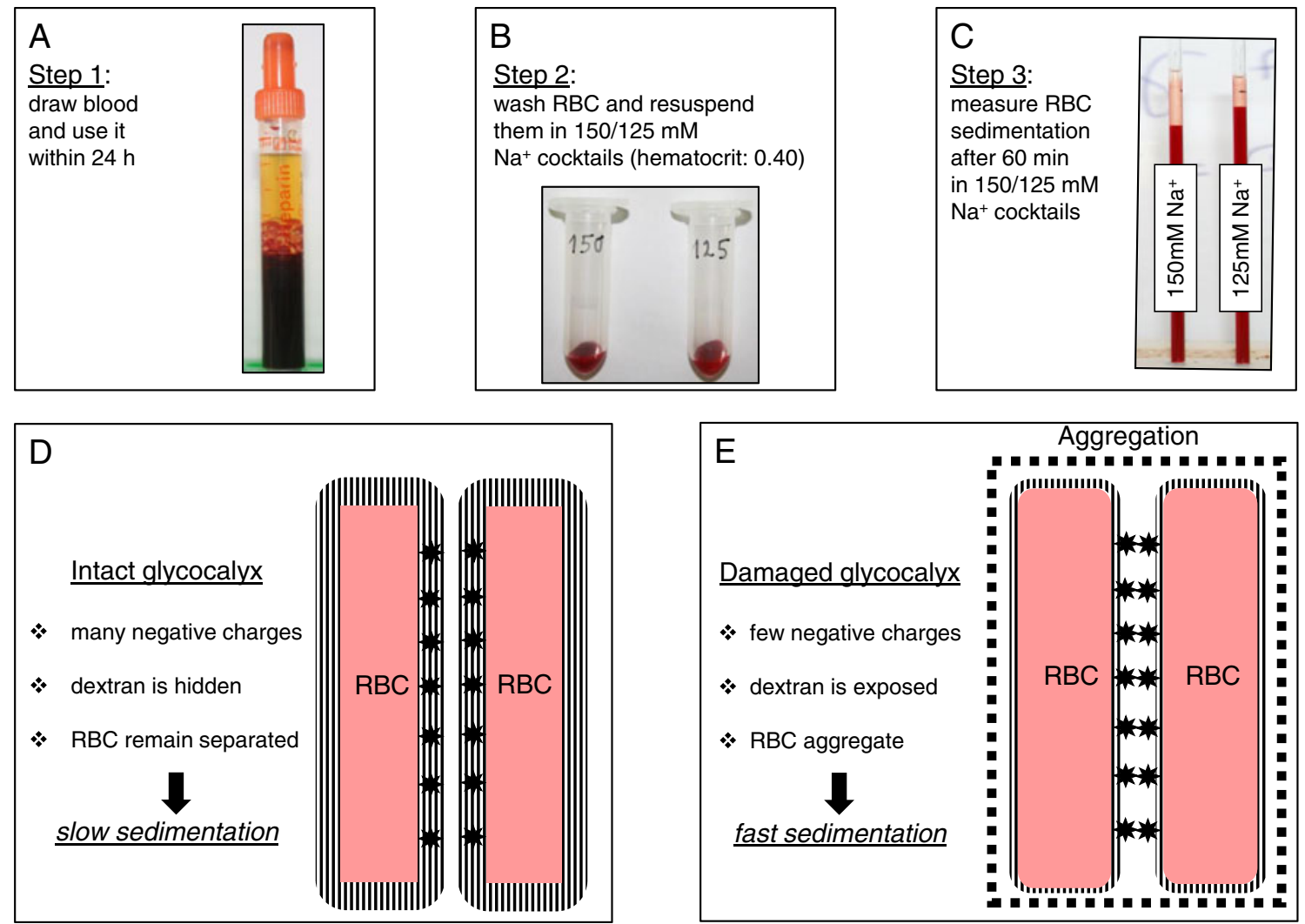

Fig. 1 Theory and practice of the salt blood test. From venipuncture to measurement $(\mathbf{a}-\mathbf{c})$. Theory underlying slow and fast erythrocyte $(\mathrm{RBC})$ sedimentation rate $(\mathbf{d}, \mathbf{e})$

undersaturated, making the test insensitive (i.e., the zeta potential is at its maximum). At too high $\mathrm{Na}^{+}$concentrations (beyond $150 \mathrm{mM}$ ), the negative charges are completely saturated again, making the test insensitive. Also, it was tried to use $\mathrm{NaCl}$ concentrations close to the physiological range. This strategy includes isosmotic conditions (appropriate addition of sucrose to the low $\mathrm{Na}^{+}$solutions) and "normal" osmolality (about $300 \mathrm{mOsm}$ ) of the electrolyte solutions. Figure 4 displays two representative measurements. From the respective supernatants, ESS could be calculated. The ESS on the left side of Fig. 4 was 2.2, indicating that $\mathrm{RBC}$ sedimentation rate was 2.2 times larger in $150 \mathrm{mM} \mathrm{Na}^{+}$than the sedimentation rate in $125 \mathrm{mM} \mathrm{Na}^{+}$. The ESS on the right side of Fig. 4 was 5.8, indicating that $\mathrm{RBC}$ sedimentation rate was 5.8 times larger in $150 \mathrm{mM}$ $\mathrm{Na}^{+}$than that in $125 \mathrm{mM} \mathrm{Na}^{+}$. Thus, ESS of blood no. 2 was more than twice as high as the ESS of blood no. 1. In a series of control experiments, the heparan sulfate residues of the RBC glycocalyx were enzymatically removed by heparinase I incubation [20], and then, ESS was measured.
Fig. 2 Time dependence of the erythrocyte sedimentation rate. $\mathrm{RBC}$ were suspended in a $150 \mathrm{mM}$ sodium cocktail (fixed hematocrit $=0.40$; see "Methods")

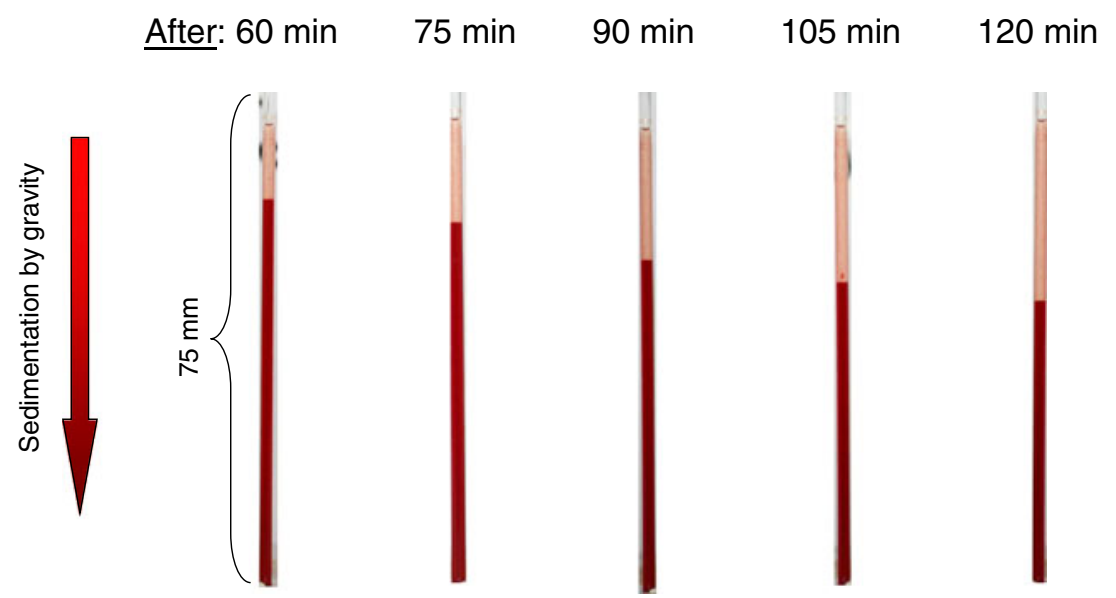




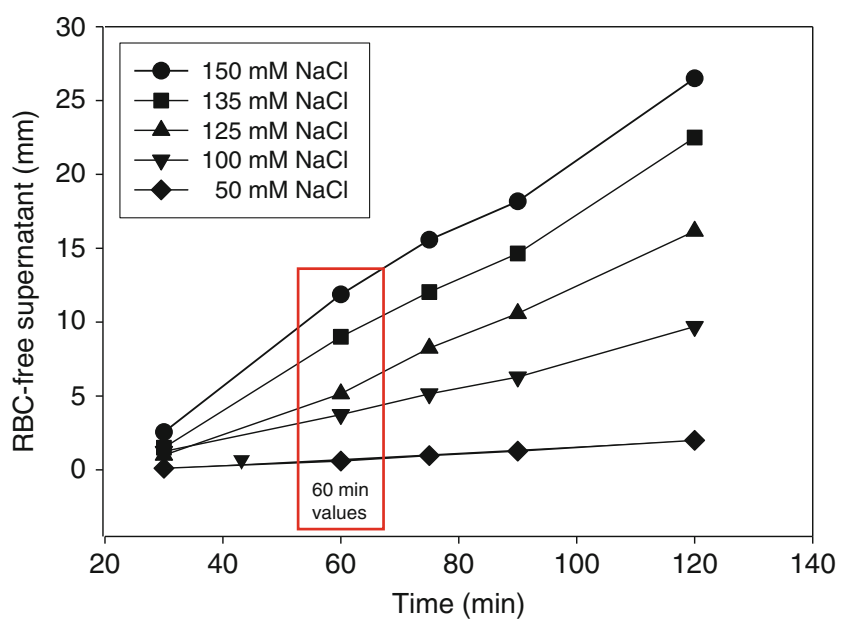

Fig. 3 Dependence of the length of the RBC-free supernatant on time and $\mathrm{NaCl}$ concentration. Osmolality is kept constant at $300 \mathrm{mOsm}$ by adding appropriate amounts of sucrose. The 60 -min value is used for evaluating the erythrocyte sodium sensitivity

In six experiments, ESS increased only by about $13 \%$ (from $2.6 \pm 0.12$ to $3.0 \pm 0.14$ ), indicating that not only heparan sulfate residues but also other negatively charged (and heparinase-insensitive) components of the glycocalyx contribute to the sodium-dependent sedimentation rate.

Figure 5 displays ESS measured in 61 healthy volunteers of similar age. The frequency distribution indicates two ESS peaks, one at about 3 and another one at about 5 . Figure 6 shows these data in more detail. Forty-six percent (28 out of 61) of the study participants exhibited an ESS of at least $20 \%$ below average. Twenty-eight percent (17 out of 61 ) of the study participants exhibited an ESS of at least $20 \%$ above average. Figure 7 shows that there is no significant gender difference of the respective ESS. It is worth mentioning here that the SBT has been performed in all experiments at a fixed hematocrit ( 0.40 ; see "Methods"). Taken together, the data indicate that there is a wide range of ESS (from 2 to 8 ) in the normal population. It should be mentioned that at least five individuals (red columns in Fig. 6) were on low-salt diets (for no obvious reason, they indicated this explicitly in a questionnaire). Remarkably, the ESS of all five of them was found in the "weakly salt-sensitive" group.

To test whether the RBC surface (i.e., the RBC glycocalyx) suffers when blood is rhythmically dragged across the surface of an endothelial monolayer and whether the expected shedding of the RBC glycocalyx could be prevented (and thereafter verified by the SBT), endothelial monolayers were incubated for 4 days with and without $10 \mu \mathrm{g} / \mathrm{ml} \mathrm{WS} 1442$, a hawthorn extract rich in polyphenols [26]. After this period of incubation, drag force treatment was applied (see "Methods"). The blood overlaying the monolayers was analyzed (performing the SBT) after a 3-h period of drag force treatment. Figure 8 shows the results. ESS analyzed from these blood samples was $5.8 \pm 0.48$ (control; $n=12$, mean \pm standard error of the mean (SEM)) and $4.5 \pm 0.31$ (WS $1442 ; n=12$, mean \pm SEM). Taken together, the erythrocyte glycocalyx is less shed (-21\%) during drag force treatment by pretreating the endothelium in culture with WS 1442. These data support previous findings, namely, that (a) WS 1442 protects the endothelial glycocalyx, and
Fig. 4 ESS measurements indicating weak erythrocyte $\mathrm{Na}^{+}$sensitivity (blood no. 1) and strong erythrocyte $\mathrm{Na}^{+}$sensitivity (blood no. 2). ESS erythrocyte sodium sensitivity
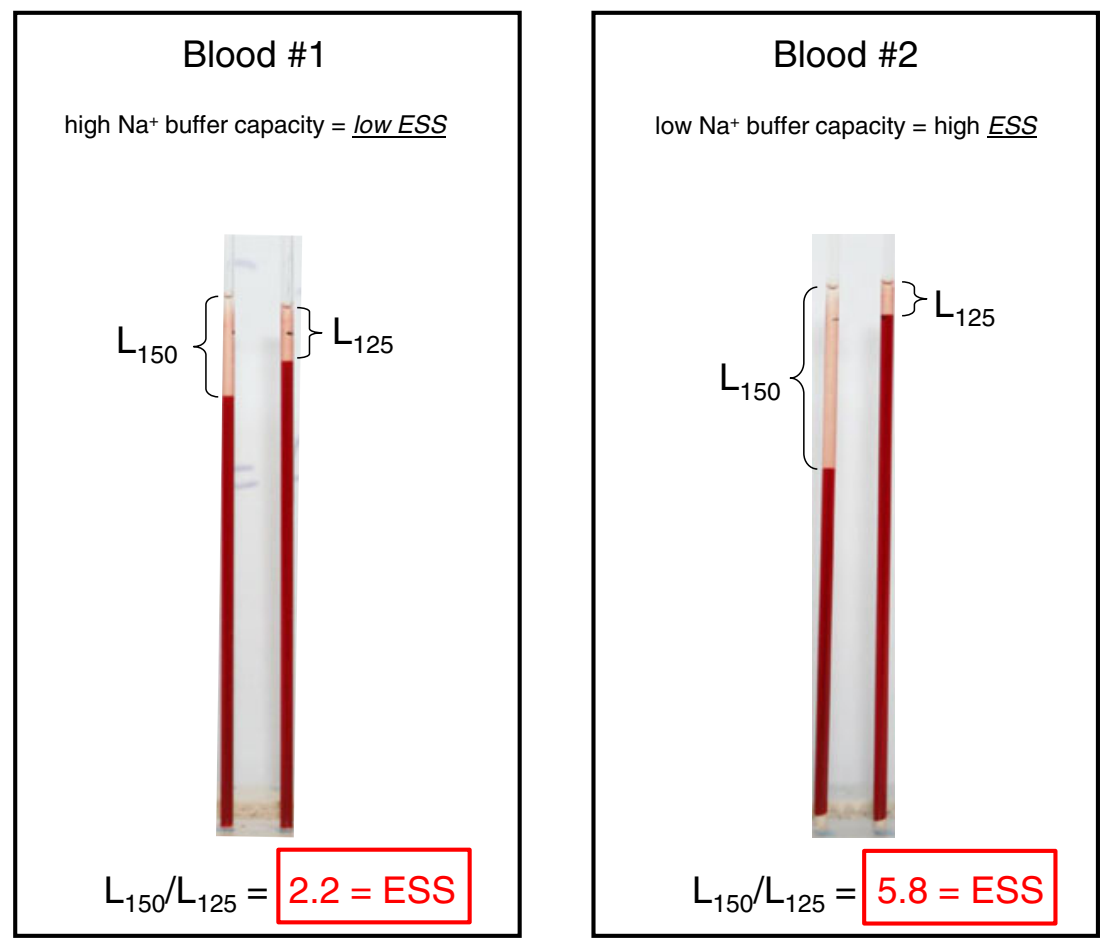
Fig. 5 Frequency distribution of the erythrocyte sodium sensitivity in a cohort of 61 study participants. Two peaks are visible

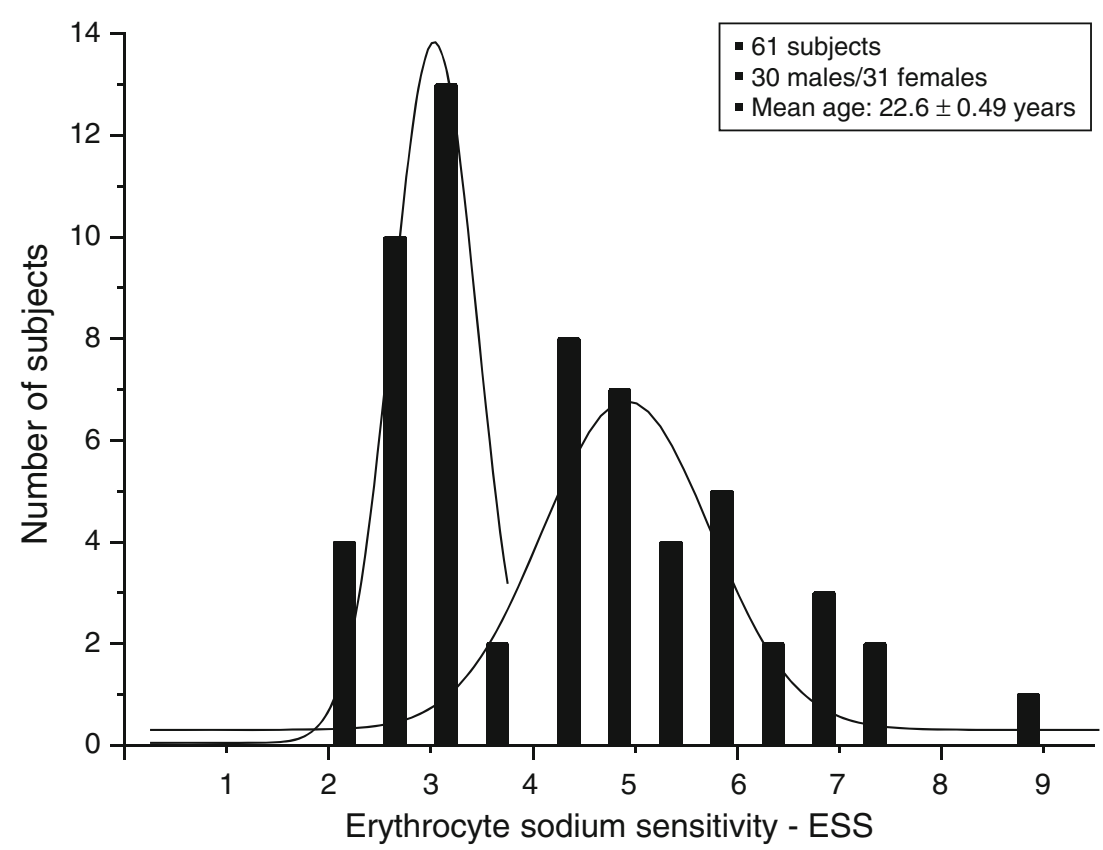

(b) the ESS reports surface properties of the endothelium with which the blood has been interacting before.

\section{Discussion}

Arterial hypertension and related vascular diseases have high mortality rates worldwide. One of the major risk factors is high sodium intake [19]. Obviously, there is an imbalance between sodium intake and renal sodium excretion. Salt sensitivity, i.e., the development of hypertension in response to salt, differs among people. The reasons for the different salt sensitivities are largely unknown. It could be related to the intrinsic ability of the kidneys to excrete sodium [7], to the limited storage capacities of the skin and muscle [14] or to a variable sodium buffer capacity of the vasculature [24]. There are reports that a single salty meal elevates plasma sodium for a few millimoles per liter which has a measureable impact on blood pressure [29] and endothelial cell function [25]. Possibly, there is a sodium sensor in vascular endothelium [23]. A candidate could be the epithelial sodium channel $(\mathrm{ENaC})$. This channel not only controls renal sodium excretion but also regulates the function of vascular endothelium [11]. Obviously, at least in vitro
Fig. 6 Erythrocyte sodium sensitivity (triple measurements) of the same study participants as displayed in Fig. 7. The overall mean value is $4.3 \pm 0.19(n=61)$. Five study participants explicitly indicated to be on a low-salt diet (red columns)

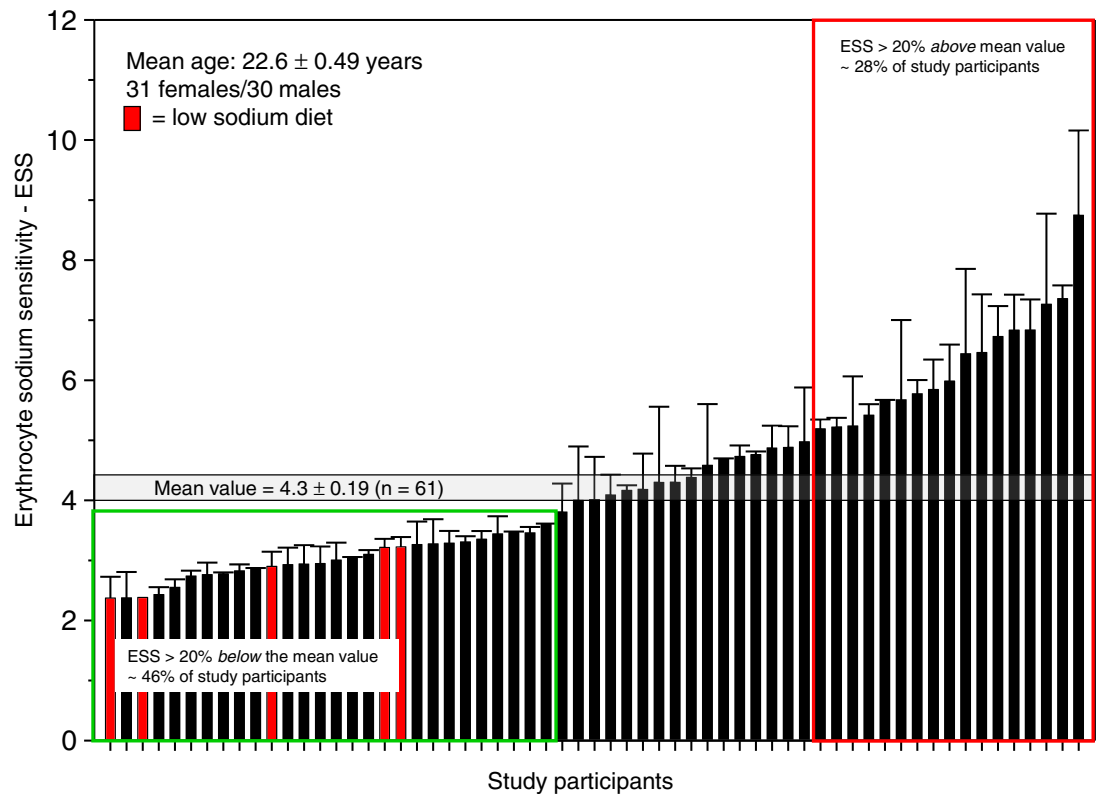


Fig. 7 Erythrocyte sodium sensitivity (triple measurements) of the same study participants as displayed in Figs. 5 and 6. Here, the data (displayed as box plots) are split into male and female groups, respectively

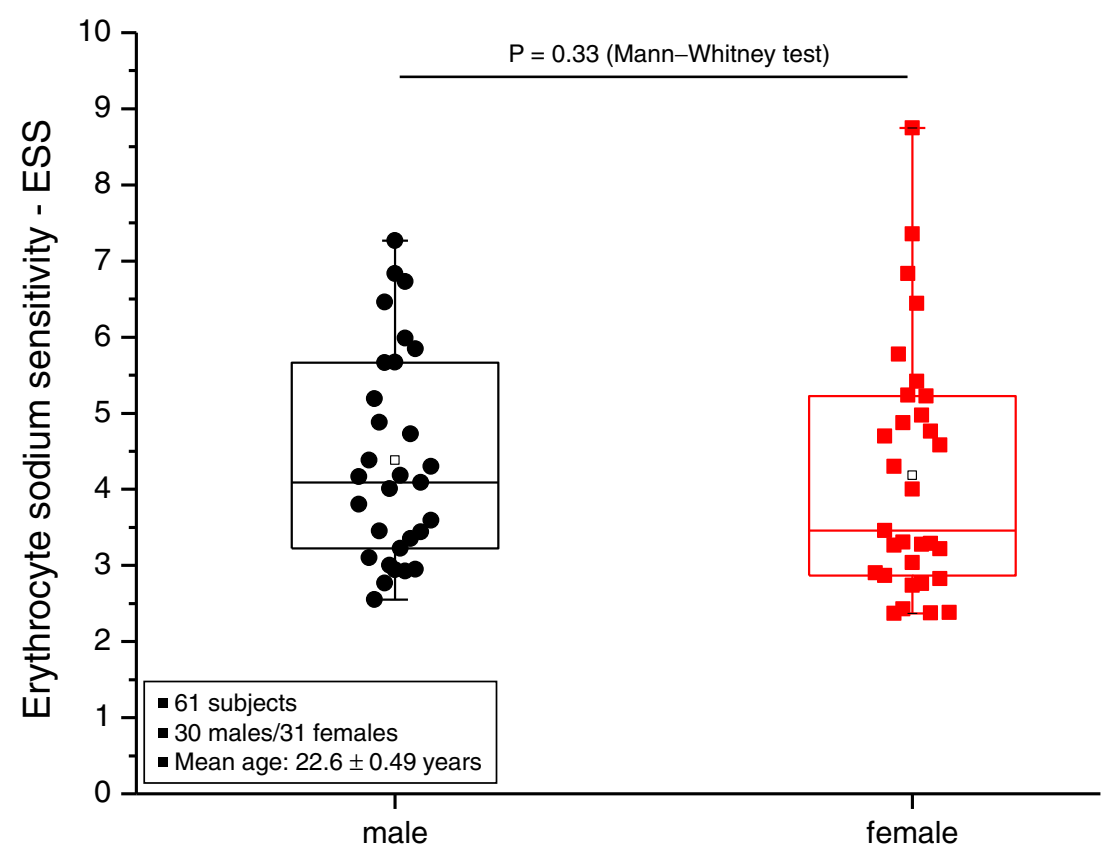

endothelial sodium sensitivity is linked to endothelial $\mathrm{ENaC}$ activity and the function of the endothelial glycocalyx [15, 24]. According to this concept, the so-called salt provocation test (SPT) was developed [21]. The SPT is an in vivo test which quantifies vascular salt sensitivity. In principle, blood pressure is measured after an oral sodium load in the presence and absence of a sodium channel blocker. The SPT evaluates the sodium buffer capacity of the endothelial glycocalyx and, hence, the vascular salt sensitivity of the individual. Although the design of the SPT is quite straightforward, two separate sessions are necessary, each with repetitive blood pressure measurements over $1 \mathrm{~h}$, an oral salt load, and the application of a sodium blocker. Therefore, it appeared attractive to develop a blood-based in vitro test system which should allow the evaluation of vascular sodium sensitivity. As described recently by means of atomic force microscopy imaging techniques, RBC "reflect" properties of the endothelial surface [20]. In detail, when heparan sulfate residues are enzymatically removed from the endothelial glycocalyx, then RBC - upon physical interaction - also lose these key glycocalyx components. This observation, namely, that the surface of RBC obviously "memorizes" the surface of the endothelium, led us to conclude that it should be sufficient to functionally test for the properties of the RBC and thus get insight into the properties of the respective endothelium.
Fig. 8 Erythrocyte sodium sensitivity measured in RBC harvested from blood overlays after drag force treatment. Control no pretreatment. WS 1442 4-day pretreatment of the endothelium (in culture) with $10 \mu \mathrm{g} / \mathrm{ml}$ of hawthorn extract WS 1442. A number of measurements are indicated in columns. The inset shows two culture flasks with endothelial monolayers and blood overlays, located on a shaker, indicating that the blood underwent drag force treatment before the SBT was performed

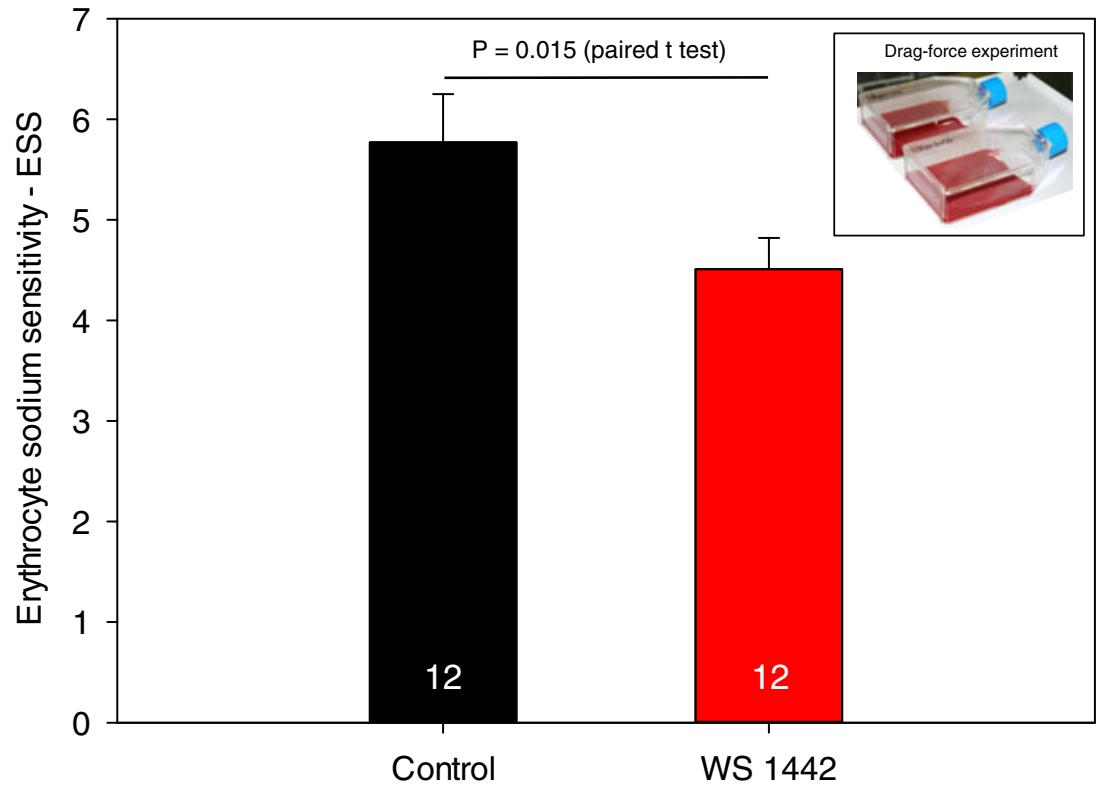


The SBT is based on the sodium-dependent RBC zeta potential $[9,10]$. Its magnitude is directly related to the negative surface charges. Besides sialic acids [5], heparan sulfate residues $[3,13,31]$ are supposed to contribute to the RBC zeta potential. They preferentially bind sodium [1]. ESR is used as a quantitative measure for the sodium-dependent zeta potential $[9,10]$. In contrast to standard ESR used as a nonspecific test for inflammatory or tumorous processes in clinical medicine [6], the SBT is performed in plasma-free electrolyte solution. Besides various concentrations of $\mathrm{NaCl}$, only sucrose (for osmolality reasons) and dextran (triggering aggregation) are present in the $\mathrm{Na}^{+}$cocktails. Therefore, the key variable in the RBC suspensions is $\mathrm{Na}^{+}$which preferentially binds to the heparan sulfate residues.

Two different $\mathrm{Na}^{+}$cocktails are sufficient for performing the SBT. The $125 \mathrm{mM} \mathrm{Na}^{+}$cocktail sets the reference value of the ESR. The $150 \mathrm{mM} \mathrm{Na}^{+}$cocktail sets the upper limit. The dimensionless ESS $\left(\mathrm{Na}^{+}{ }_{150} / \mathrm{Na}^{+}{ }_{125}\right)$ is a measure for the salt sensitivity of an individual. It would have the value "1" when the increase of $\mathrm{Na}^{+}$from 125 to $150 \mathrm{mM}$ would not change the zeta potential at all. This would indicate "no sodium sensitivity," a theoretical value that will never occur. A value above 1 indicates "sodium sensitivity," i.e., as larger is this value as more sensitive to sodium is the individual's vasculatory system (i.e., RBC and endothelium).

One of the aims of this study was to provide, as a first step, a "reference value" of sodium sensitivity expressed as ESS. Therefore, we performed the SBT in a cohort of 61 healthy young individuals. Although the number of study participants is rather low and thus the interpretation of the data is limited, there are two peaks in ESS. This could not only be due to a genetic predisposition [28] but also due to distinct differences in lifestyle [8]. Possibly, the left peak includes individuals with a "weak" sensitivity to sodium, while the right peak includes individuals with a "marked" sensitivity to sodium (see Fig. 5). Gender differences cannot explain these two peaks.

The SBT will gain a medical relevance only if the concept, namely, that the sodium buffer capacity of the endothelial glycocalyx (related to the inverse of ESS) can be indeed evaluated, is proven, at least in an in vitro experiment. This led us to perform "drag force" experiments in combination with the vasoprotective compound WS 1442. This Crataegus extract is known to improve endothelial function [2] and specifically interacts with the endothelial glycocalyx. At least in vitro, WS 1442 has been shown to unfold ("swell") the glycocalyx and lowers endothelial sodium permeability [26]. Indeed, ESS significantly decreased by $21 \%$ when the endothelium was pretreated for several days with this compound. Taken together, we assume a mechanism as follows:
Treatment of the endothelium with WS 1442 in vitro swells the endothelial glycocalyx [26]. This improves electrical surface negativity and, thus, endothelial sodium buffer capacity [24]. During drag force treatment, erythrocytes mechanically interact. By these means, the RBC surfaces "acquire" properties of the endothelial surface, i.e., their sodium-dependent zeta potentials rise. This results in a decrease of ESS.

Although this is only a first step in the proof of this concept, it opens some interesting perspectives. Sodium sensitivity, at the level of the vascular system, is probably not a "constant" parameter of an individual but is rather a variable that can be (negatively or positively) influenced. This view could make the SBT as a potentially valuable test system in the control of medical measures undertaken to improve vascular function.

Acknowledgments This work was supported by the Deutsche Forschungsgemeinschaft (Koselleck grant OB 63/18). I gratefully acknowledge the networking activities of COST Action TD 1002. The experimental protocol was approved by the local Ethics Committee (Ärztekammer Westfalen-Lippe, approval number 2012-029-f-S). The SBT is covered by pending patent no.: EP12194442.

Conflict of interest The author declares that he has no conflict of interest.

Open Access This article is distributed under the terms of the Creative Commons Attribution License which permits any use, distribution, and reproduction in any medium, provided the original author(s) and the source are credited.

\section{References}

1. Bevan JA (1993) Flow regulation of vascular tone. Its sensitivity to changes in sodium and calcium. Hypertension 22:273-281

2. Brixius K, Willms S, Napp A, Tossios P, Ladage D, Bloch W, Mehlhorn U, Schwinger RH (2006) Crataegus special extract WS 1442 induces an endothelium-dependent, NO-mediated vasorelaxation via eNOS-phosphorylation at serine 1177. Cardiovasc Drugs Ther 20:177-184

3. Drzeniek Z, Stocker G, Siebertz B, Just U, Schroeder T, Ostertag W, Haubeck HD (1999) Heparan sulfate proteoglycan expression is induced during early erythroid differentiation of multipotent hematopoietic stem cells. Blood 93:2884-2897

4. Edgell CJ, Haizlip JE, Bagnell CR, Packenham JP, Harrison P, Wilbourn B, Madden VJ (1990) Endothelium specific WeibelPalade bodies in a continuous human cell line, EA.hy926. In Vitro Cell Dev Biol 26:1167-1172

5. Eylar EH, Madoff MA, Brody OV, Oncley JL (1962) The contribution of sialic acid to the surface charge of the erythrocyte. J Biol Chem 237:1992-2000

6. Grzybowski A, Sak J (2012) A short history of the discovery of the erythrocyte sedimentation rate. Int J Lab Hematol 34:442-444

7. Guyton AC (1991) Blood pressure control-special role of the kidneys and body fluids. Science 252:1813-1816

8. Hoffmann IS, Alfieri AB, Cubeddu LX (2007) Effects of lifestyle changes and metformin on salt sensitivity and nitric oxide metabolism in obese salt-sensitive Hispanics. J Hum Hypertens 21:571-578 
9. Jan KM, Chien S (1973) Influence of the ionic composition of fluid medium on red cell aggregation. J Gen Physiol 61:655-668

10. Jan KM, Chien S (1973) Role of surface electric charge in red blood cell interactions. J Gen Physiol 61:638-654

11. Jeggle P, Callies C, Tarjus A, Fassot C, Fels J, Oberleithner H, Jaisser F, Kusche-Vihrog K (2013) Epithelial sodium channel stiffens the vascular endothelium in vitro and in liddle mice. Hypertension 61(5):1053-1059

12. Kleinewietfeld M, Manzel A, Titze J, Kvakan H, Yosef N, Linker RA, Muller DN, Hafler DA (2013) Sodium chloride drives autoimmune disease by the induction of pathogenic T17 cells. Nature 496(7446):518-522

13. Kobayashi K, Kato K, Sugi T, Takemae H, Pandey K, Gong H, Tohya Y, Akashi H (2010) Plasmodium falciparum BAEBL binds to heparan sulfate proteoglycans on the human erythrocyte surface. J Biol Chem 285:1716-1725

14. Kopp C, Linz P, Dahlmann A, Hammon M, Jantsch J, Muller DN, Schmieder RE, Cavallaro A, Eckardt KU, Uder M, Luft FC, Titze J (2013) 23Na magnetic resonance imaging-determined tissue sodium in healthy subjects and hypertensive patients. Hypertension 61:635-640

15. Korte S, Wiesinger A, Straeter AS, Peters W, Oberleithner H, KuscheVihrog K (2012) Firewall function of the endothelial glycocalyx in the regulation of sodium homeostasis. Pflugers Arch 463:269-278

16. Kusche-Vihrog K, Oberleithner H (2012) An emerging concept of vascular salt sensitivity. F1000. Biol Rep 4:20

17. Kusche-Vihrog K, Sobczak K, Bangel N, Wilhelmi M, Nechyporuk-Zloy V, Schwab A, Schillers H, Oberleithner H (2008) Aldosterone and amiloride alter $\mathrm{ENaC}$ abundance in vascular endothelium. Pflugers Arch 455:849-857

18. Kusche-Vihrog K, Urbanova K, Blanque A, Wilhelmi M, Schillers H, Kliche K, Pavenstadt H, Brand E, Oberleithner H (2011) Creactive protein makes human endothelium stiff and tight. Hypertension 57:231-237

19. Meneton P, Jeunemaitre X, de Wardener HE, MacGregor GA (2005) Links between dietary salt intake, renal salt handling, blood pressure, and cardiovascular diseases. Physiol Rev 85:679-715
20. Oberleithner H (2013) Vascular endothelium leaves fingerprints on the surface of erythrocytes. Pflügers Arch. doi:10.1007/s00424013-1288-y

21. Oberleithner H (2012) A physiological concept unmasking vascular salt sensitivity in man. Pflugers Arch 464:287-293

22. Oberleithner H (2012) Two barriers for sodium in vascular endothelium? Ann Med 44(Suppl 1):S143-S148

23. Oberleithner H, Kusche-Vihrog K, Schillers H (2010) Endothelial cells as vascular salt sensors. Kidney Int 77:490-494

24. Oberleithner H, Peters W, Kusche-Vihrog K, Korte S, Schillers H, Kliche K, Oberleithner K (2011) Salt overload damages the glycocalyx sodium barrier of vascular endothelium. Pflugers Arch 462:519-528

25. Oberleithner H, Riethmuller C, Schillers H, MacGregor GA, de Wardener HE, Hausberg M (2007) Plasma sodium stiffens vascular endothelium and reduces nitric oxide release. Proc Natl Acad Sci U S A 104:16281-16286

26. Peters W, Drueppel V, Kusche-Vihrog K, Schubert C, Oberleithner H (2012) Nanomechanics and sodium permeability of endothelial surface layer modulated by hawthorn extract WS 1442. PLoS One 7:e29972

27. Pribush A, Hatskelzon L, Meyerstein N (2011) A novel approach for assessments of erythrocyte sedimentation rate. Int J Lab Hematol 33:251-257

28. Sanada H, Jones JE, Jose PA (2011) Genetics of salt-sensitive hypertension. Curr Hypertens Rep 13:55-66

29. Suckling RJ, He FJ, Markandu ND, MacGregor GA (2012) Dietary salt influences postprandial plasma sodium concentration and systolic blood pressure. Kidney Int 81:407-411

30. Titze J, Lang R, Ilies C, Schwind KH, Kirsch KA, Dietsch P, Luft FC, Hilgers KF (2003) Osmotically inactive skin $\mathrm{Na}^{+}$storage in rats. Am J Physiol Renal Physiol 285:F1108-F1117

31. Vogt AM, Winter G, Wahlgren M, Spillmann D (2004) Heparan sulphate identified on human erythrocytes: a Plasmodium falciparum receptor. Biochem J 381:593-597

32. Weinberger MH (1996) Salt sensitivity of blood pressure in humans. Hypertension 27:481-490 